FORMATION Formation emploi

Revue française de sciences sociales

142 | Avril-Juin 2018

Génération 2010 : diversité des parcours de réussite

\title{
Trajectoires résidentielles et professionnelles des jeunes : quand l'accès à l'emploi ne signe pas la décohabitation
}

Residential and occupational trajectories of young people: When access to employment does not sign "not living with parents"

Wohn- und Berufsverläufe junger Menschen : wenn der Zugang zur

Beschäftigung nicht das Verlassen des Elternhauses bedeutet

Trayectorias residenciales y profesionales de los jóvenes : cuando el acceso al empleo no marca el fin de la cohabitación

\section{Philippe Cordazzo}

\section{OpenEdition}

Journals

Édition électronique

URL : http://journals.openedition.org/formationemploi/5499

DOI : 10.4000/formationemploi.5499

ISSN : 2107-0946

Éditeur

La Documentation française

Édition imprimée

Date de publication : 23 août 2018

Pagination : 167-185

ISSN : 0759-6340

Référence électronique

Philippe Cordazzo, «Trajectoires résidentielles et professionnelles des jeunes : quand l'accès à l'emploi ne signe pas la décohabitation », Formation emploi [En ligne], 142 | Avril-Juin 2018, mis en ligne le 23 août 2020, consulté le 30 octobre 2020. URL : http://journals.openedition.org/formationemploi/ 5499 ; DOl : https://doi.org/10.4000/formationemploi.5499

(c) Tous droits réservés 


\title{
Trajectoires résidentielles et professionnelles des jeunes : quand l'accès à l'emploi ne signe pas la décohabitation
}

\begin{abstract}
PhILIPPE CORDAZZO
Professeur de démographie à l'université de Strasbourg et membre de I'UMR-7363 SAGE (Sociétés, Acteurs, Gouvernement en Europe). II s'intéresse plus particulièrement aux " parcours étudiants, de la formation à l'nsertion professionnelle » (Titre de son HDR), notamment par le prisme des vulnérabilités et de la transition à l'âge adulte

Résumé

Trajectoires résidentielles et professionnelles des jeunes : quand l'accès à l'emploi ne signe pas la décohabitation

Selon les résultats de l'enquête Génération 2010 du Céreq, 57,4\% des jeunes adultes ayant arrêté leurs études à vingt ans ont connu, au cours des trois années suivantes, une trajectoire professionnelle durable d'accès immédiat ou rapide à l'emploi. Mais ils sont aussi près de $56 \%$ à loger encore chez leurs parents trois ans après. L'analyse présentée ici combine les deux types de trajectoires professionnelles et résidentielles. Elle montre que quitter le foyer parental n'est pas nécessairement synonyme d'émancipation financière et que l'accès à l'emploi n'est pas davantage synonyme de décohabitation systématique.
\end{abstract}

Mots clés : jeune ; trajectoire d'insertion ; condition de vie ; enquête d'insertion

Abstract

Residential and occupational trajectories of young people: When access to employment does not sign "not living with parents"

According to the results of Cereq Génération 2010 survey, $57.4 \%$ of young adults who left school in the last twenty years have lived, over the next three years, a sustainable professional trajectory of immediate or rapid access to employment. But, they are also close to $56 \%$ to stay at home with their parents three years later. The analysis presented here combines the two types of occupational and residential trajectories and shows that leaving the parental home coincident not necessarily with financial emancipation and that access to employment is not synonymous with systematic housing situation of "not living with parents".

Keywords: young person ; pathway to integration ; living condition ; school-to-work transition survey

Journal of Economic Literature: J 12 ; J 24.

Traduction : Auteur. 
Avec la fin des études, une nouvelle étape de la transition vers l'âge adulte est franchie, et déjà une autre est en perspective, soit l'entrée durable sur le marché du travail. Ce processus de transition connaît une double évolution. Une "désynchronisation " ${ }^{1}$ des étapes de la transition vers l'âge adulte (Galland, 2011), et un processus d'entrée dans l'âge adulte de plus en plus lent qui s'accompagne de difficultés d'insertion. Cette dernière est, par ailleurs, de plus en plus retardée, notamment du fait de l'allongement des études (en raison de la course vers un niveau plus élevé, première protection contre le chômage, voire de l'obtention de plusieurs diplômes pour maximiser les chances d'embauche).

Or, les jeunes connaissent aujourd'hui des parcours d'accès à la vie adulte de plus en plus complexes, dans un contexte de crise économique qui les touche plus particulièrement (Van-de-Velde, 2013). Cette situation économique défavorable entraîne une augmentation des périodes d'inactivé et de chômage pouvant affecter les trajectoires d'autonomie résidentielle. Les données des enquêtes Emploi de l'Institut national de la statistique et des études économiques (Insee) ${ }^{2}$ montrent un taux d'emploi en diminution sur la période 2010-2013, de 62,4 \% à 59,0 \% pour les titulaires d'un bac +2 , de 31,3\% à 29,8\% pour les titulaires d'un baccalauréat ou d'un brevet professionnel, et de $20,8 \%$ à $17,8 \%$ pour les non-diplômés. De plus, la crise a eu des conséquences sur l'emploi des jeunes de 20-24 ans, faisant passer leur taux d'emploi de 48,8 \% en 2012, à 46,8 \% en 2013 (Insee) ${ }^{3}$.

Mais la crise ne peut expliquer à elle seule le départ ou le non-départ du foyer parental. Ainsi, d'autres facteurs comme le coût financier de l'accès à un logement autonome, la capacité à mobiliser une aide financière de la famille et des aides publiques (Portela \& al., 2014), ou encore la mise en couple, vont jouer un rôle socialement différencié sur l'autonomie résidentielle (Castel \& al., 2016). Ainsi, certains jeunes adultes vont trouver certaines contreparties "financières " à une cohabitation prolongée avec leurs parents et inversement, d'autres vont privilégier l'autonomie résidentielle (avec ou sans aide des parents), malgré le surcoût budgétaire.

Cet article s'intéresse donc aux trajectoires d'entrée dans la vie adulte, et plus particulièrement à la relation complexe entre la trajectoire professionnelle et la trajectoire résidentielle. Il s'inscrit dans la continuité des travaux de Virginie Mora \& al. (2008) et de Cécile Van De Velde (2015). Son propos sera de questionner la réalité des liens entre décohabitation et emploi ou au contraire entre vie chez les parents et chômage, mais aussi entre vie chez les parents et emploi. L'accès à l'emploi durable favorise-t-il toujours la décohabitation?

1. Pour le sociologue Olivier Galland (2011), la "désynchronisation » signifie que les différentes phases du passage à l'âge adulte, soit la fin des études, la décohabitation du foyer parental, l'entrée durable sur le marché du travail, ou encore la mise en couple et la maternité, ne sont plus des "séquences " qui se succèdent dans un ordre prédéfini, notamment avec un départ du foyer parental de plus en plus temporaire.

2. Source : Insee, enquête Emploi 2010, séries longues.

3. Id. 
À l'inverse, une situation de chômage durable est-elle encore synonyme de maintien au domicile parental?

Les travaux présentés dans cet article se distinguent de ceux précédemment cités, par les méthodes utilisées, afin d'apporter un éclairage complémentaire et original. Ainsi, la méthode utilisée pour l'analyse des trajectoires est "l'analyse des séquences ", qui a pour objectif principal "d'identifier - dans la diversité d'un corpus de séquences constituées de séries d'états successifs - les régularités, les ressemblances, puis, le plus souvent, de construire des typologies de "séquences-types". L'analyse de séquences constitue donc un moyen de décrire, mais aussi de mieux comprendre le déroulement de divers processus" (Robette, 2012). Dans cet article, la méthode est utilisée en combinant de manière simultanée les deux phénomènes étudiés, à savoir l'insertion professionnelle et l'autonomie résidentielle. Cela permet de mesurer la fréquence des combinaisons entre ces différentes situations professionnelles et résidentielles.

Notre première partie présente et discute des apports et limites des choix opérés et des méthodes utilisées. Elle justifie le choix d'une application originale de l'analyse des séquences, mais aussi celui de travailler sur les jeunes sortant du système scolaire à l'âge de vingt ans (100191 jeunes dans l'enquête Génération 2010 après affectation des poids). Par la suite, l'évolution de l'autonomie résidentielle et de l'insertion professionnelle des jeunes adultes, sortis du système scolaire à vingt ans, sera d'abord analysée à un niveau global. Dans une dernière partie, les déterminants des différentes trajectoires d'entrée dans la vie adulte (insertion professionnelle et autonomie résidentielle) seront examinés. À cet effet, nous mobiliserons la méthode de l'analyse des séquences pour identifier des trajectoires-types, puis nous utiliserons une analyse mesurant les facteurs qui influencent la probabilité de connaître une autre trajectoire que la trajectoire la plus fréquente.

\section{Données et méthodes pour reconstituer les trajectoires}

Nous exposons ici en quoi l'utilisation des données de l'enquête Génération est pertinente pour la reconstitution de trajectoires individuelles par l'analyse des séquences. Ensuite, nous discutons des avantages et limites d'un travail sur les seuls sortants du système scolaire à l'âge de vingt ans. 


\subsection{Identifier les trajectoires types}

\subsubsection{Un calendrier mensuel pour reconstituer les trajectoires}

Une des spécificités des enquêtes Générations est la mise en place d'un calendrier qui décrit, mois par mois, la situation des jeunes. Il permet de construire des typologies à partir de la description de ces situations. Ces typologies offrent une vision synthétique des premières années sur le marché du travail, soit les trajectoires d'accès rapide à l'emploi, d'accès différé à l'emploi, de décrochage, etc. (Rouaud \& Joseph, 2014). En plus du calendrier professionnel, il existe aussi un calendrier du mode de cohabitation. Il collecte les états suivants : vit chez les parents, vit en couple hors de chez les parents ${ }^{4}$, vit seul. On croise les deux calendriers afin de mettre en relation les trajectoires professionnelles et résidentielles, c'est-à-dire de confronter le calendrier des différentes étapes du passage à l'âge adulte, à savoir l'entrée sur le marché du travail et la décohabitation. Cette comparaison peut notamment permettre de mesurer l'impact éventuel des trajectoires de vulnérabilisation économique sur la re-cohabitation. Nous nous référons ici aux travaux de Mora, Sulzer, Goffette \& Joseph, de 2008, pour compléter notre approche.

L'enquête Génération 2010 du Céreq, auprès des sortants du système scolaire, permet d'analyser les parcours, en mettant en avant l'étude des transitions ou de s'attacher aux "trajectoires types" (Lelièvre, 2009). Pour mieux appréhender l'enchaînement des différents événements dans le temps, le choix s'est porté sur la construction de trajectoires professionnelles et de décohabitation pour la population des sortants du système scolaire à vingt ans.

\subsubsection{L'analyse des séquences : une méthode pour reconstituer les trajectoires}

Une manière d'analyser le lien entre les trajectoires professionnelles et résidentielles des jeunes à la sortie des études supérieures est d'utiliser l'analyse des séquences. Il ne s'agit plus seulement d'essayer d'expliquer les trajectoires, mais de décrire précisément, par une analyse statistique, les trajectoires types. L'appariement optimal (analyse des séquences) est central dans le corpus des méthodes descriptives d'analyses des séquences (Robette \& Thibault, 2008). Ces dernières sont particulièrement appropriées pour traiter de trajectoires biographiques, notamment pour l'étude de la transition vers l'âge adulte (Billari, 2001), ou des carrières professionnelles. Pour ce faire, nous utilisons le logiciel R, qui propose actuellement la méthode la plus complète et la plus puissante en matière d'analyse de séquences 5 .

4. La situation " vivre chez les parents " est prioritaire par rapport à la situation, "vivre en couple "; ainsi, un jeune qui vit en couple chez ses parents sera enregistré comme vivant chez les parents.

5. Pour l'analyse des séquences sur R : Package principal utilisé, TraMineR, pour la méthode, voir Nicolas Robette, 2012 \& Matthias Studer, 2012. 
Nous avons suivi les principes fondamentaux de la méthode (Billari 2001 ; Robette, op. cit.) et fait les choix nécessaires à sa réalisation :

Unité d'observation (mois), soit trente états qui composent la trajectoire. Si le choix de l'unité temporelle, à savoir mensuelle, peut être discuté, il semble pertinent par rapport à l'étude des trajectoires professionnelles des jeunes. En effet, si d'un côté l'unité mensuelle pose le problème du questionnement rétrospectif qui donne lieu à une surestimation de l'immobilité et à une sous-estimation du chômage (Rémillon, 2016), elle apporte, en revanche, un gain en termes de précision ;

- $\quad$ Pour la description de la distribution des états, nous avons utilisé la mesure de la distance moyenne au centre de la classe et celle de l'entropie de Shanon comme indicateur du degré d'homogénéité de la trajectoire ;

- La méthode de l'appariement optimal consiste, pour chaque paire de séquences, à compter le nombre minimal de modifications (substitutions, suppressions, insertions) qu'il faut faire subir à l'une des séquences pour obtenir l'autre. Pour ce travail, les coûts de substitution sont dérivés des probabilités de transition entre les éléments. Ainsi, le coût de substitution entre deux éléments est d'autant plus élevé que la probabilité de transition entre ces éléments est faible.

Afin de mieux comprendre l'agencement des trajectoires professionnelles et d'autonomie résidentielle, nous avons réalisé une analyse des séquences en couplant temporellement les événements des deux trajectoires (Figure 1). Nous avons choisi quatre états pour l'insertion professionnelle (Emploi, chômage, études et inactivité) et avons gardé les trois états de la situation de l'habitat (vivre chez les parents, vivre en couple hors du domicile des parents et vivre seul). Nous avons ainsi douze états possibles après croisement des situations professionnelles et résidentielles.

Figure 1. Couples d'évènements de la trajectoire professionnelle et résidentielle

\begin{tabular}{|l|l|l|}
\hline Etats & & \\
\hline Vivre en couple et être au chômage & Vivre chez les parents et être au chômage & Vivre seul(e) et être au chômage \\
\hline Vivre en couple et être en emploi & Vivre chez les parents et être en emploi & Vivre seul(e) et être en emploi \\
\hline Vivre en couple et être en études & Vivre chez les parents et être en études & Vivre seul(e) et être et en études \\
\hline Vivre en couple et être inactif & Vivre chez les parents et être inactif & Vivre seul(e) et être inactif \\
\hline
\end{tabular}

Source : Auteur.

\subsection{Choix de la population : les moins de vingt ans}

Pour étudier le lien entre les trajectoires d'autonomie résidentielle et d'insertion professionnelle, nous avons choisi de travailler sur les sortants du système scolaire à vingt ans. Cela répond à des critères méthodologiques et pose certaines limites.

En travaillant sur la génération ayant eu vingt ans à la fin de ses études, nous répondons au critère d'homogénéité de constitution d'une cohorte. C'est-à-dire avoir connu un même 
événement (fin des études), à un même moment (2010) et à âge identique (vingt ans). De plus, le critère de l'âge permet d'enlever un obstacle important qui est celui de la dépendance du phénomène étudié avec l'âge. En effet, plusieurs travaux sur l'autonomie résidentielle montrent que la décohabitation augmente avec l'avancée en âge (RégnierLoilier, 2011), Ainsi, en choisissant comme population étudiée les jeunes sortants du système éducatif à vingt ans, nous disposons d'une cohorte homogène au regard de la date de survenue de l'événement constitutif de la cohorte et d'un suivi (de trois ans, donc de vingt à vingt-trois ans) englobant l'âge moyen (vingt ans) au moment du départ du foyer parental. Ce choix méthodologique met la focale sur les jeunes qui partent précocement du foyer parental. Cependant, il devrait aussi surestimer la décohabitation des femmes qui partent, de manière générale, un an et demi à deux ans plus tôt que les hommes.

Néanmoins, ce choix a des conséquences sur la population étudiée qui, par construction de l'échantillon, est sélectionnée selon le niveau de diplôme. Ainsi, si nous avons de jeunes adultes diplômés à différents niveaux d'études, ils sont fortement concentrés sur le niveau baccalauréat. 9,7\% terminent leurs études avec le baccalauréat général, 12,4\% avec un baccalauréat technologique et 32,1\% munis d'un baccalauréat professionnel (Tableau 3). De plus, les diplômés du supérieur sont principalement des diplômés de BTS (brevet de technicien supérieur). Les travaux de Sophie Orange (2013) ont montré la spécificité des parcours de ces jeunes, souvent issus des catégories populaires.

En outre, et de manière mécanique, choisir les jeunes de vingt ans va influer sur le taux de décohabitation. Ainsi, avec une population plus jeune, le taux de décohabitation serait plus faible, et inversement, avec une population plus âgée, il serait plus élevé. Si l'on se réfere aux travaux de Portela \& Denezaire (2014) sur la Génération 2007 en 2010, les taux de décohabitation varient en fonction de l'âge à la fin des études, de $32 \%$ pour les 19 ans et moins, à $47 \%$ pour les $20-21$ ans et $73 \%$ pour les $22-23$ ans. Ainsi, en étudiant les jeunes de vingt ans et en suivant l'évolution de leur autonomie résidentielle jusqu'à l'âge de vingt-trois ans, nous avons un spectre d'analyse intéressant. En effet, la période de vingt à vingt-trois ans apparaît comme charnière dans le début de la marche progressive vers l'indépendance résidentielle.

\section{L'autonomie résidentielle : une évolution différenciée dans un contexte économique dégradé}

Nous nous intéressons ici à l'évolution de l'autonomie résidentielle et de l'insertion professionnelle. Nous mobilisons des données de cadrage des jeunes adultes sortis du système scolaire à vingt ans, dont une première analyse "globale " des liens éventuels entre autonomie résidentielle et insertion professionnelle. 


\subsection{Un accès à l'autonomie résidentielle plus rapide pour les femmes et qui augmente avec l'âge}

Pour les sortants du système scolaire en 2010, à vingt ans, les résultats confirment que l'autonomie résidentielle augmente avec l'avancée en âge pour les femmes et les hommes (Figure 2). De plus, les femmes partent du foyer parental et se mettent en couple, en moyenne deux ans plus tôt que les hommes. Ainsi, dix-huit mois après la fin des études, c'est-à-dire à vingt et un an et demi, $40 \%$ des femmes ont décohabité, contre $30 \%$ des hommes (Rault \& Régnier-Loilier, 2015). Ce départ à des âges plus avancés et l'accès à l'autonomie résidentielle ont aussi été observés, pour la Génération 2007, en 2010 (Portela \& Dezenaire 2014). Ces phénomènes et leurs durées s'inscrivent dans la crise économique, et plus particulièrement comme conséquence de la crise du logement dans les territoires (Driant, 2016), tout en gardant un caractère différencié socialement (Castell $\&$ al., 2016). Il est donc prévisible de retrouver ces données sur le long terme, tant que la crise continue, avec les mêmes impacts, plus ou moins ressentis, suivant l'origine sociale des étudiants.

Figure 2. Evolution de la décohabitation des personnes ayant arrêté leurs études à 20 ans

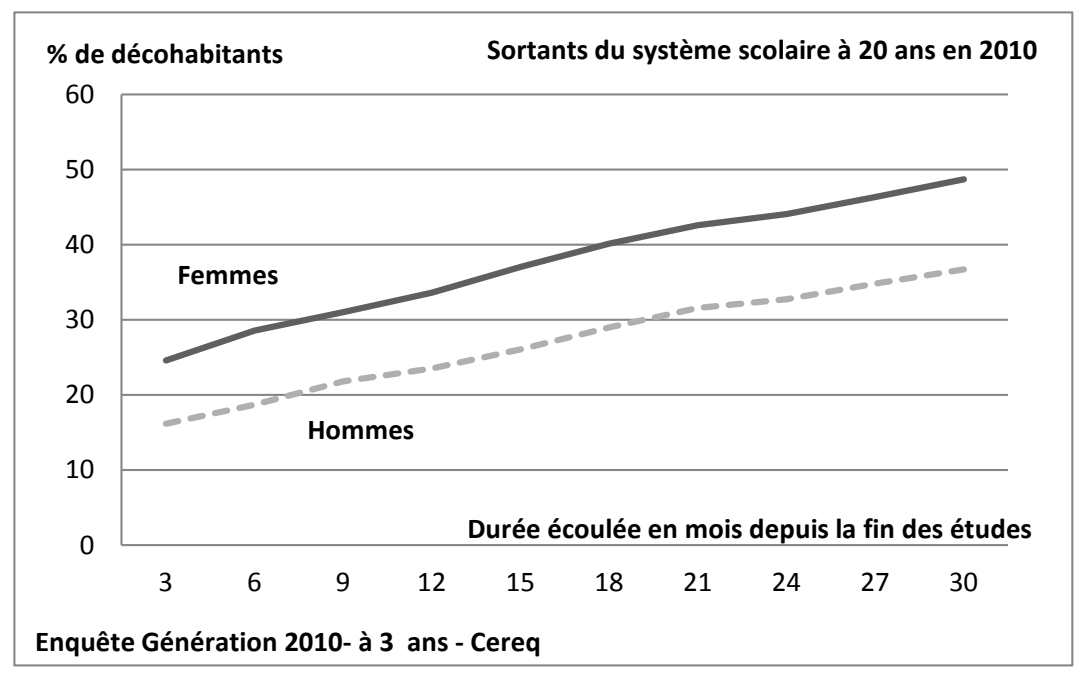

Champ : Personnes ayant arrêté leurs études à 20 ans. Lecture : dix-huit mois après la fin des études, $40 \%$ des femmes vivent en dehors du foyer parental, seules ou en couple.

Source : Centre d'études et de recherche sur les qualifications (Céreq), Enquêtes Génération 2010 à trois ans. 


\subsection{Une insertion professionnelle difficile dans un contexte dégradé}

Les sortants du système scolaire en 2010, à vingt ans, sont dans une situation contrastée en termes d'insertion professionnelle (Figure 3). Tout d'abord, l'insertion professionnelle des hommes est toujours supérieure à celle des femmes, pour chaque durée, et suit une évolution parallèle. Mais si les taux d'insertion professionnelle augmentent rapidement pour atteindre, douze mois après, $80 \%$ des hommes en emploi et $76 \%$ des femmes, on observe, sur les douze mois suivants, un ralentissement de la croissance, voire une stagnation. Surtout, à partir du vingt-quatrième mois après la fin des études, le taux d'insertion professionnelle diminue pour, à trente mois, revenir à son niveau atteint douze mois après la fin des études. Ce phénomène semble fortement lié à une dégradation du marché du travail sur la période étudiée. Ainsi, pour les 20-24 ans, le taux d'emploi est passé de $48,8 \%$ en 2010 , à $46,8 \%$ en $2013^{6}$, diminuant de manière semblable pour les hommes ( $52,1 \%$ à $50,4 \%)$ et les femmes ( $45,5 \%$ à 43,3 \%).

Figure 3. Evolution de l'insertion professionnelle des personnes ayant arrêté leurs études à 20 ans

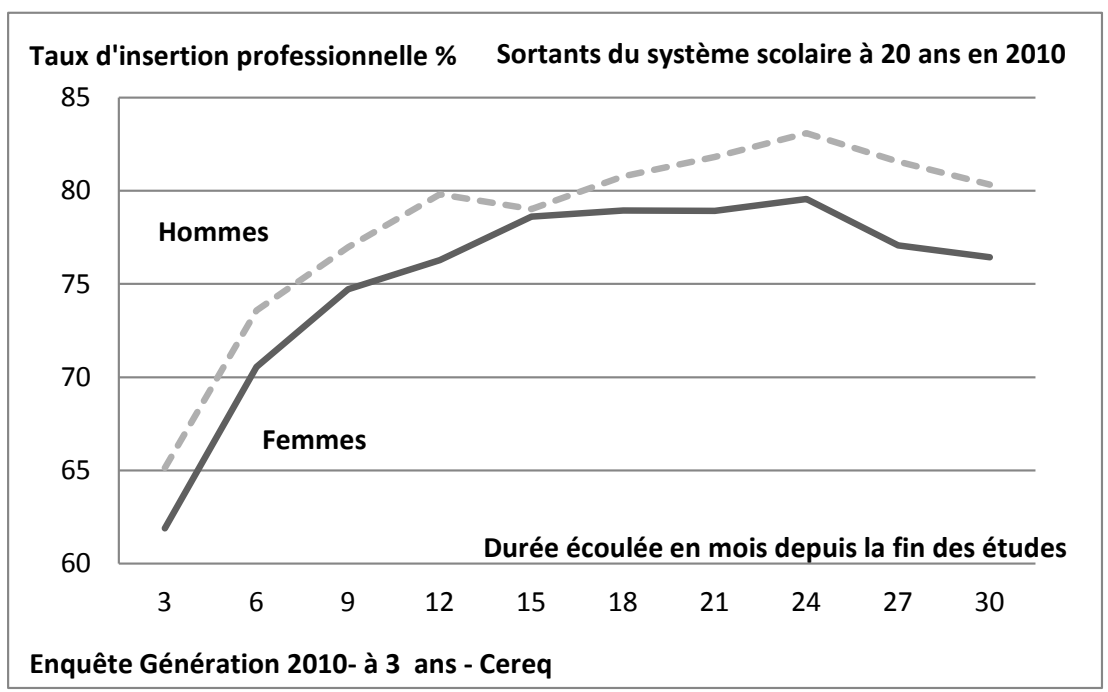

Champ : Personnes ayant arrêté leurs études à vingt ans. Lecture : neuf mois après la fin des études, le taux d'insertion professionnelle (nombre de personnes en emploi rapporté aux personnes en emploi ou en recherche d'emploi) des femmes est de $75 \%$.

Source : Centre d'études et de recherche sur les qualifications (CEREQ), Céreq, Enquêtes Génération 2010 à trois ans.

6. Source : Insee, enquêtes Emploi (calculs Insee). 
Le taux d'insertion se définit comme la part des diplômés occupant un emploi, quel qu’il soit, sur l'ensemble des diplômés qui sont sur le marché du travail. Il est calculé sur les diplômés français issus de la formation initiale, entrés immédiatement et durablement sur le marché de l'emploi après l'obtention de leur diplôme.

\subsection{Cohabitation avec les parents et accès à l'emploi durable prédominent}

Dans cette partie et la suivante, les trajectoires sont analysées en fonction de la fréquence d'apparition et selon le calendrier d'accès à l'autonomie résidentielle ou à l'emploi. Pour le calendrier professionnel, trois états sont distingués : immédiat (dès la fin des études), rapide (au cours des six premiers mois après la fin des études) et différé (entre six et dix-huit mois après la fin des études).

Nous disposons d'un calendrier résidentiel qui collecte les états suivants de l'autonomie résidentielle : vit chez les parents, vit en couple hors de chez ses parents, vit seul. Nous avons réalisé une analyse des séquences (cf. Annexe en version électronique de l'article) qui nous a permis de distinguer cinq grands types de trajectoires (Tableau 1, en colonne) : départ différé en couple (HAB.1), départ différé seul (HAB.5), vit chez les parents (HAB.2), vit en couple (HAB.4), vit seul (HAB.3). Si la marche vers l'indépendance des jeunes ayant arrêté leurs études à vingt ans est progressive, elle est plus marquée pour certains d'entre eux. Ainsi, trente mois après, 44,3\% sont en situation d'autonomie résidentielle (21,4\% seuls et $22,9 \%$ en couple).

Nous avons retenu neuf types de trajectoires professionnelles ${ }^{7}$ (Tableau 1, en ligne). La trajectoire la plus fréquente $(43,8 \%)$ est celle d'accès immédiat et durable à l'emploi, suivie de celle relative à l'accès rapide et durable à l'emploi (13,6\%). Ainsi, les jeunes adultes sortis du système scolaire à vingt ans ont majoritairement des trajectoires professionnelles d'accès durable à l'emploi (57,4\%). La fréquence de ces trajectoires est différenciée selon le sexe. Ainsi, les femmes sorties du système scolaire à vingt ans ont moins souvent des trajectoires d'accès immédiat à l'emploi que les hommes (41\% contre $46,4 \%$ ).

De plus, nous l'avons vu précédemment (Figure 3), la situation professionnelle des jeunes adultes sortis du système scolaire à vingt ans commence à se stabiliser, voire à se dégrader dix-huit mois après la fin des études, conséquence, semble-t-il, d'un marché du travail défavorable. Cela se retrouve dans deux types de trajectoires : celle des jeunes adultes qui connaissent une sortie de l'emploi vers le chômage (6,8 \%) ou l'inactivité

7. Ces trajectoires sont issues de la variable TYPOTRAJ de l'enquête Génération, qui décrit les trajectoires professionnelles des sortants du système scolaire à partir du calendrier mensuel. Cette variable résulte d'une typologie des trajectoires (méthode du LIRHE) prenant en compte comme états sur le marché du travail : emploi, chômage, inactivité et formations ou reprises études. De plus, elle prend en compte l'évolution des états mensuellement. 
$(1,9 \%)$ et celle de chômage durable ou récurrent $(9,1 \%)$. De plus, une proportion non négligeable des jeunes est dans une situation d'alternance entre recherche d'emploi et reprise d'études $(11 \%)$, peut-être comme solution à leur précarité professionnelle ou pour se redonner une chance de trouver un emploi.

Cette présentation distincte des trajectoires résidentielles et professionnelles révèle une prédominance des situations où les jeunes adultes vivent chez leurs parents et où ils sont en emploi durable. Il s'agit de mesurer maintenant dans quelle mesure ces différentes situations se combinent ou non. Les résultats du Tableau 1 nous permettent d'obtenir une première mesure globale de la combinaison des trajectoires résidentielles et professionnelles. La lecture des résultats par les trajectoires résidentielles a été privilégiée.

La trajectoire la plus fréquente $(60,3 \%)$ de jeunes ayant arrêté leurs études à vingt ans concerne ceux qui vivent chez leurs parents au cours des trente mois (HAB.2). S'ils accèdent en majorité durablement à l'emploi, de manière immédiate ou rapide, ils sont proportionnellement un peu moins nombreux que l'ensemble de la population étudiée $(53,1 \%$ contre $57,4 \%)$. C'est encore plus marquant pour les jeunes adultes ayant quitté le foyer parental de manière différée pour vivre seuls (49,2\%). Cela pourrait s'expliquer par une moindre urgence à trouver un travail quand on peut rester chez les parents, ou quand la mise en couple n'entre pas en jeu pour motiver la recherche de l'autonomie financière.

Nous pouvons distinguer deux types de trajectoires avec une autonomie résidentielle pour vivre en couple hors du foyer parental, selon que les jeunes le quittent rapidement et durablement (12,3\%-HAB.4) ou de manière différée (6,3\%-HAB.1). Les jeunes adultes qui connaissent ce type de trajectoire résidentielle sont proportionnellement plus nombreux que l'ensemble de la population étudiée $(63,9 \%$ pour la trajectoire HAB. 1 et $66,8 \%$ pour la trajectoire HAB.4, contre $57,4 \%$ pour l'ensemble) à accéder de manière durable (immédiate ou rapide) à l'emploi. C'est aussi le cas (HAB.3) des jeunes adultes partis immédiatement ou rapidement du foyer parental pour vivre seuls $(67,7 \%)$. 
Tableau 1. Trajectoires résidentielles et professionnelles

\begin{tabular}{|l|c|c|c|c|c|c|}
\cline { 2 - 7 } \multicolumn{1}{c|}{} & \multicolumn{6}{c|}{ Types de trajectoires résidentielles* } \\
\hline Types de trajectoires professionnelles & $\begin{array}{c}\text { HAB.1 } \\
\mathbf{( 6 , 3} \%)\end{array}$ & $\begin{array}{c}\text { HAB.2 } \\
\mathbf{( 6 0 , 3} \%)\end{array}$ & $\begin{array}{c}\text { HAB.3 } \\
\mathbf{( 1 5 , 2} \%)\end{array}$ & $\begin{array}{c}\text { HAB.4 } \\
\mathbf{( 1 2 , 3} \%)\end{array}$ & $\begin{array}{c}\text { HAB.5 } \\
\mathbf{( 5 , 9} \%)\end{array}$ & Total \\
\hline Accès immédiat et durable à l'emploi & 47,4 & 39,6 & 56,4 & 51,3 & 35,7 & 43,8 \\
\hline Accès rapide et durable à l'emploi & 16,5 & 13,5 & 11,3 & 15,5 & 13,5 & 13,6 \\
\hline Accès progressif à l'emploi après chômage & 8,6 & 8,4 & 4,4 & 7,4 & 15,7 & 8,1 \\
\hline Accès progressif à l'emploi après inactivité & 2,6 & 3,2 & 4,1 & 2,7 & 6,5 & 3,4 \\
\hline Sortie d'emploi vers le chômage & 7,7 & 7,2 & 4,6 & 6,9 & 7,3 & 6,8 \\
\hline $\begin{array}{l}\text { Sortie temporaire du marché du travail vers } \\
\text { l'inactivité }\end{array}$ & 2,3 & 1,7 & 2,7 & 2,3 & 0,4 & 1,9 \\
\hline Chômage durable ou récurrent & 4,8 & 11,9 & 6,0 & 4,7 & 2,9 & 9,1 \\
\hline Inactivité durable & 0,9 & 2,6 & 1,4 & 2,5 & 0,8 & 2,2 \\
\hline $\begin{array}{l}\text { Périodes importantes ou récurrentes de RE } \\
\text { et formations en cours de parcours }\end{array}$ & 9,1 & 11,9 & 9,2 & 6,6 & 17,4 & 11,0 \\
\hline Total & 100 & 100 & 100 & 100 & 100 & 100 \\
\hline
\end{tabular}

*Types de trajectoires résidentielles : HAB.1 (départ différé du foyer parental et vie en couple); HAB.2 (vit chez les parents) ; HAB.3 (vit seul) ; HAB.4 (vit en couple) ; HAB.5 (départ différé du foyer parental et vit seul).

Lecture : Parmi les personnes qui connaissent la trajectoire HAB.2 (Vit chez les parents), 39,6\% ont connu un accès immédiat et durable à l'emploi.

Source : Centre d'études et de recherche sur les qualifications (Céreq), Céreq, Enquêtes Génération 2010 à trois ans.

À ce stade de l'analyse, la stabilité dans l'emploi favorise la décohabitation, que ce soit en couple ou seul. Ceux qui restent chez leurs parents ont une trajectoire d'emploi moins stable ou y accèdent moins vite.

\section{Des trajectoires entre insertion professionnelle et autonomie résidentielle}

Dans cette dernière partie, nous verrons qu'il est relativement fréquent de rester chez ses parents, malgré l'accès à l'emploi, et nous reviendrons sur les huit trajectoires repérées.

\subsection{Vivre chez les parents et être en emploi, une situation relativement fréquente}

Comme expliqué précédemment, nous avons combiné mensuellement les situations résidentielles (vivre chez les parents, vivre en couple hors du domicile parental et vivre seul) et professionnelles (Emploi, chômage, études ou inactivité).

La combinaison la plus fréquente $(44,5 \%)$ est celle où le jeune adulte vit chez ses parents tout en étant en emploi (Figure 5). Trois autres situations combinées apparaissent dans plus 
de $10 \%$ des cas : vivre chez les parents et être au chômage (15,9\%); vivre en couple et être en emploi $(11,9 \%)$; vivre seul et être en emploi $(11,4 \%)$.

Ainsi, nous voyons qu'il n'y a aucune systématicité entre une situation d'emploi et l'autonomie résidentielle. À l'inverse, c'est même la combinaison « vivre chez les parents et être en emploi » qui est la plus fréquente. Lanalyse des trajectoires individuelles devrait nous permettre de mieux comprendre ce phénomène.

Figure 4. Fréquence (\%) des différentes combinaisons de situations professionnelles et résidentielles pour l'ensemble des trajectoires

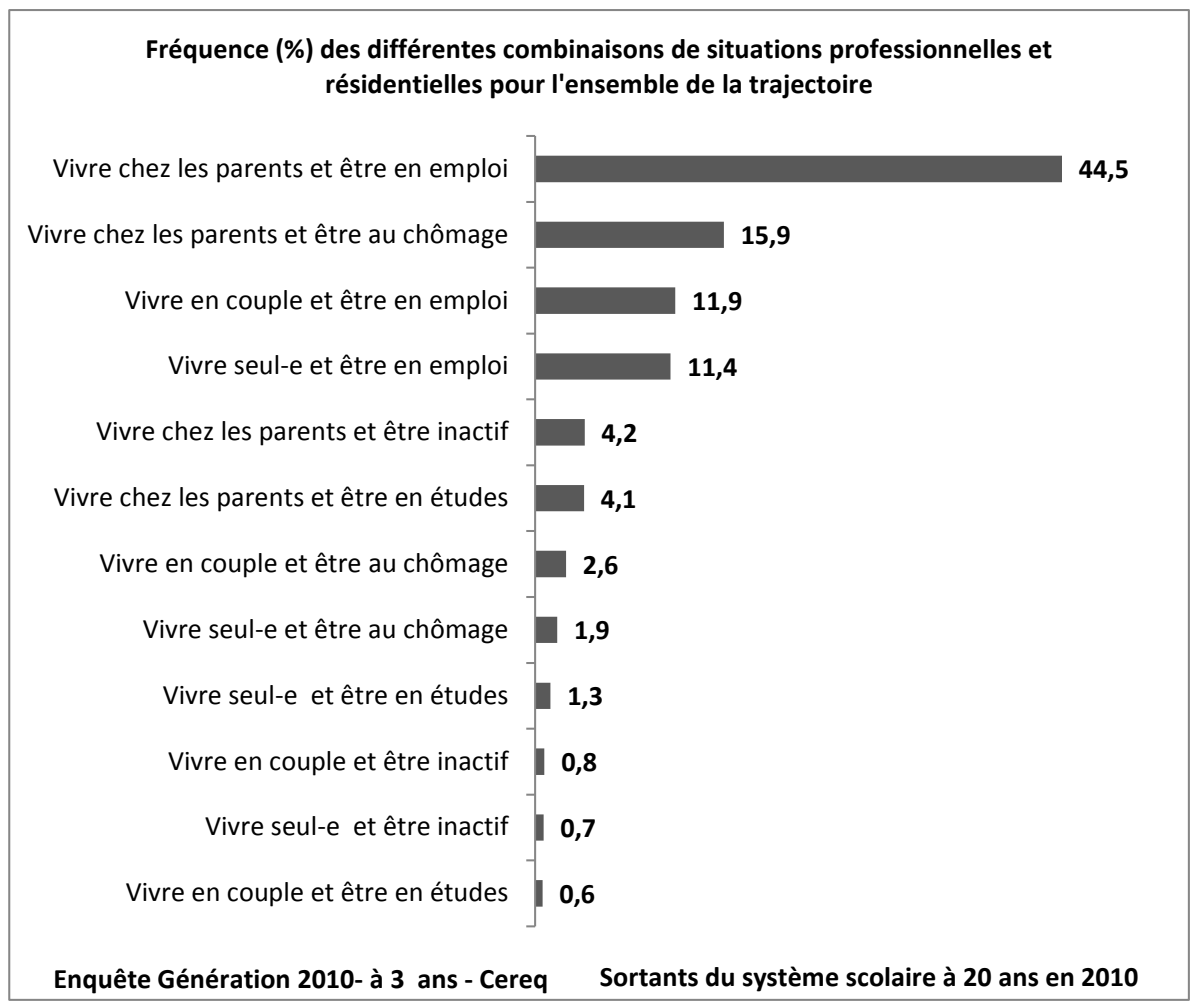

Champ : Personnes ayant arrêté leurs études à vingt ans.

Lecture : La combinaison situation professionnelle et résidentielle la plus fréquente concerne " vivre chez les parents et être en emploi » dans $44,5 \%$ des cas.

Source : Centre d'études et de recherche sur les qualifications (CEREQ), Céreq, Enquêtes Génération 2010 à trois ans.

\subsection{Huit trajectoires d'entrée dans la vie adulte}

L'analyse des séquences sur les sortants du système scolaire en 2010, à vingt ans, a permis de distinguer huit types de trajectoires (Tableau 2). La partie suivante va décrire leurs trajectoires professionnelles et de marche progressive vers l'indépendance. 
Nous obtenons huit trajectoires professionnelles et d'autonomie résidentielle, différenciées en fonction du calendrier de survenue des événements (départ du foyer parental, accès à l'emploi, etc.). $75 \%$ des jeunes adultes connaissent des trajectoires professionnelles (Trajectoires : 1, 3, 5, 6, 7) correspondant à l'obtention d'un emploi, de manière rapide ou différée. Majoritairement, ces jeunes adultes vivent encore chez leurs parents. C'est aussi le cas des trajectoires marquées par le chômage ou l'inactivité (Trajectoires : 2, 4 et 8).

Une des difficultés dans la constitution d'une typologie, comme c'est le cas avec l'analyse des séquences, est l'obtention d'un équilibre entre homogénéité des trajectoires au sein des classes, hétérogénéité des trajectoires entre classes et représentativité statistique des classes. Nous avons ainsi huit trajectoires qui permettent de respecter globalement ces exigences, notamment avec une lisibilité des combinaisons entre la situation professionnelle et résidentielle. Les courbes d'entropie montrent que les trajectoires sont homogènes sur la durée, faisant apparaître un peu d'hétérogénéité en début et fin d'observation pour les trajectoires 1 et 6 , en début pour la trajectoire 7 , à la fin pour la trajectoire 8 , et en cours, pendant les périodes de transition entre états pour les trajectoires 3 et 5 . La trajectoire 4 est hétérogène par rapport à la situation professionnelle, mais homogène en termes d'autonomie résidentielle. La trajectoire 2 est la plus hétérogène, notamment en termes d'autonomie résidentielle. Mais finalement, elle reste relativement lisible par rapport aux formes des trajectoires des individus marqués par la précarité professionnelle, quelle que soit leur trajectoire d'autonomie résidentielle.

Tableau 2. Trajectoires professionnelles et résidentielles

\begin{tabular}{|l|c|}
\multicolumn{1}{|c|}{ Typologie trajectoire professionnelle et résidentielle } & Fréquence par modalité \\
& \multicolumn{1}{|c|}{ (\%) } \\
\hline Trajectoire 1: Vit chez les parents et accès durable à l'emploi & 41,0 \\
\hline Trajectoire 2: Précarité et hétérogénéité de l'autonomie résidentielle & 9,8 \\
\hline Trajectoire 3 : Accès rapide à l'emploi et départ différé du foyer parental pour vivre en couple & 4,7 \\
\hline Trajectoire 4:Vit chez les parents et inactivité ou études & 6,0 \\
\hline Trajectoire 5: Accès rapide à l'emploi et départ différé du foyer parental pour vivre seul & 7,6 \\
\hline Trajectoire 6: Accès immédiat ou rapide à l'emploi et vit seul & 10,3 \\
\hline Trajectoire 7: Accès immédiat ou rapide à l'emploi et vit en couple & 11,5 \\
\hline Trajectoire 8: Vit chez les parents et chômage & 9,1 \\
\hline
\end{tabular}

Champ : 4133 répondants représentatifs de 100191 sortants en 2010 du système scolaire à vingt ans. Obtention des trajectoires par l'analyse des séquences avec le logiciel $R$ (TraMineR).

Source : Céreq, Enquête Génération 2010 à 3 ans. 
Nous allons maintenant analyser les facteurs déterminants pour les trajectoires d'entrée dans la vie adulte (Tableau 3, en annexe de la version électronique de l'article). Pour cela, nous avons réalisé une régression logistique multinomiale et comparons notamment des rapports de chances entre eux.

\section{Encadré 1. Description de la méthode de régression logistique multinomiale}

La régression logistique multinomiale permet de comparer la probabilité de connaître une trajectoire $(2,3,4,5,6,7$, ou 8) plutôt que la trajectoire la plus fréquente (Trajectoire $1:$ Vivre chez les parents avec un accès immédiat ou rapide à l'emploi), en fonction des variables introduites dans le modèle.

Dans cet article, nous calculons et comparons des odds ratio ou " rapports de chances relatives » entre eux. "L'odd ratio » est un rapport (ratio) entre les chances relatives (odds) des uns et des autres. Dans notre analyse, il s'agit de mesurer la chance relative de connaître l'une des trajectoires $(2,3,4,5,6,7$ ou 8) plutôt que la trajectoire la plus fréquente ( 1 - vivre chez les parents et être en emploi). Nous faisons ainsi le rapport entre les chances relatives des femmes de connaître l'une des trajectoires $(2,3,4,5,6,7$ ou 8$)$ plutôt que la trajectoire la plus fréquente ( 1 - vivre chez les parents et être en emploi) et celles des hommes, ou encore le même rapport entre les chances relatives entre les enfants d'ouvriers et les enfants de cadres. À titre d'exemple (Tableau 3, en annexe de la version électronique de l'article) les femmes ont un odd ratio de 1,95 pour la trajectoire 2. Ainsi, elles ont 1,95 fois plus de chances de connaître la trajectoire 2 plutôt que la trajectoire 1, que les hommes. Pour une réflexion sur les odds ratio, voir Pierre Mercklé, Les " odds ratios » sont-ils la meilleure façon de mesurer des inégalités, quanti.hypotheses.org.

Les variables déterminantes suivantes ont été mobilisées : sexe, niveau de diplôme, lieu de naissance des parents, position professionnelle des parents et lieu de résidence. En revanche, les variables couple, enfants, et mobilité résidentielle n'ont pas été introduites dans les modèles, car trop fortement dépendantes du phénomène étudié.

\subsubsection{Rester chez les parents, malgré un accès rapide et durable à l'emploi (41\%)}

La première trajectoire, la plus fréquente $(41 \%)$, concerne des jeunes qui, très majoritairement, ont accédé à l'emploi immédiatement ou rapidement, et durablement, tout en restant vivre chez leurs parents. Nous constatons alors que l'accès à l'emploi durable n'a pas coïncidé avec une autonomie résidentielle. Compte tenu de la jeunesse de ce public, il ne s'agit pour autant pas de parler de phénomène "Tanguy ${ }^{8}$.

Les jeunes adultes qui connaissent cette trajectoire ne se distinguent pas de l'ensemble du public, si ce n'est que ce sont plus souvent des hommes $(58,5 \%$ contre $52,7 \%$ pour l'ensemble), qui ont terminé leurs études en Ile-de-France (19,9\% contre 15,4\%) et qui résidaient, au moment de l'enquête, en Ile-de-France (19,4\% contre 16,1\%).

8. Le phénomène "Tanguy " désigne un phénomène social selon lequel certains jeunes adultes tardent à partir du foyer parental, ou en tous cas le font plus tardivement que la moyenne. Or, l'âge moyen à la décohabitation est de 23 ans et nous suivons une population à des âges antérieurs (de 20 à 23 ans). 
Pour comprendre les raisons qui font que ces jeunes, qui ont un accès rapide et durable à l'emploi, ne connaissent pas une autonomie résidentielle, on peut avancer une hypothèse liée au coût de l'accès à un logement autonome. En effet, malgré une situation d'emploi, le budget de ces jeunes et les capacités financières des parents ne leur permettent pas d'accéder à l'autonomie résidentielle. Ces trajectoires, "formes de cohabitation prolongée, relèvent davantage de situations subies que choisies. Et elles ne sont pas nécessairement synonymes de dépendance financière " (Van de Velde, 2008).

\subsubsection{Décohabitation pour vivre seul et accès immédiat ou rapide et durable à l'emploi $(17,9 \%)$}

Deux trajectoires composent ce groupe et se distinguent par le calendrier de la décohabitation pour vivre seul. D'un côté, les jeunes adultes partis progressivement du foyer parental (trajectoire $5-7,6 \%$ ) et ceux partis immédiatement ou rapidement (trajectoire $6-10,3 \%)$.

Ce qui différencie principalement les jeunes adultes ayant connu l'une ou l'autre de ces trajectoires est le calendrier de départ du foyer parental. Ainsi, dans la trajectoire 6 (Accès immédiat ou rapide à l'emploi et vit seul) le départ du foyer parental s'est fait dans les trois mois suivant la fin des études (cf. Figure 5 en annexe de la version électronique de l'article), tandis que dans la trajectoire 5 (Accès rapide à l'emploi et départ différé du foyer parental pour vivre seul), les départs se répartissent progressivement entre six mois et vingt-quatre mois après la fin des études. Pour cette dernière trajectoire, on note qu'une partie des jeunes adultes a connu un accès un peu plus tardif à l'emploi, entre six mois et douze mois après la fin des études (cf. Figure 5, en annexe de la version électronique de l'article). Ces jeunes adultes ont pour caractéristiques communes d'être moins fréquemment : des femmes, issues de l'immigration et ayant fait leurs études en Ile-de-France. En revanche, les jeunes adultes qui ont obtenu un baccalauréat général ou un diplôme du supérieur, et ceux résidant, trois ans après, en Ile-de-France, ont une probabilité plus importante de connaître l'une de ces trajectoires.

En schématisant, le profil type de ces jeunes adultes est celui d'un jeune homme, diplômé du baccalauréat général ou du supérieur court, fils d'artisan, commerçant, ou de chef d'entreprise, ayant suivi des études en dehors de la région Ile-de-France et qui est parti en Ile-de-France où il a trouvé du travail.

\subsubsection{Décohabitation pour vivre en couple et accès rapide à l'emploi (16,2 \%)}

Deux trajectoires composent ce groupe et se distinguent par le calendrier de la décohabitation pour vivre en couple. D’un côté, les jeunes adultes partis progressivement du foyer parental (trajectoire $3-4,7 \%$ ) et de l'autre, ceux partis immédiatement ou rapidement (trajectoire $7-11,5 \%$ ). Ce qui différencie principalement les jeunes adultes ayant connu l'une ou l'autre de ces trajectoires est le calendrier de départ du foyer parental. Ainsi, dans la trajectoire 7 , le départ du foyer parental s'est opéré dans les neuf mois suivant la fin des 
études (Figure 5, en annexe de la version électronique de l'article), tandis que dans la trajectoire 3, les départs se répartissent de manière relativement équilibrée entre douze mois et vingt-quatre mois après la fin des études.

Ces jeunes adultes ont pour caractéristique commune d'être plus fréquemment des femmes (odds ratio $=3,10$ (trajectoire 7 ) et 1,75 (trajectoire 3)). Cette surreprésentation des femmes est en grande partie liée au fait qu'elles se mettent en couple deux ans en moyenne plus tôt que les hommes (Jauneau, 2007). De plus, ce sont souvent des parents, puisque $18,4 \%$ des jeunes adultes ayant connu la trajectoire 3 et 25,8 \% de ceux ayant suivi la trajectoire 7 ont ou vont avoir des enfants.

\subsubsection{Précarité des trajectoires et transition à l'âge adulte fortement retardée $(24,9 \%)$}

Trois trajectoires sont caractéristiques de ce groupe, celle de jeunes plus ou moins précaires qui restent vivre chez les parents et qui reprennent des études au bout d'une année (trajectoire $4-6,0 \%$ ), celle de jeunes qui restent vivre chez les parents en étant au chômage (trajectoire $8-9,1 \%$ ) et enfin celle de jeunes marqués par des trajectoires de précarité (trajectoire $2-9,8 \%$ ).

La trajectoire 4 concerne plus fréquemment des jeunes qui sont sans diplôme (odds ratio= 4,20 ), ont obtenu un baccalauréat général (odds ratio $=2,67$ ) ou un baccalauréat technologique (odds ratio $=3,93$ ). Rappelons, comme un des éléments explicatifs, que pour les jeunes adultes sans diplôme (public cible des politiques d'insertion) et, dans une moindre mesure, pour les diplômés d'un baccalauréat général, le recours probable à une formation professionnelle est la solution envisagée pour sortir d'une situation de chômage durable ou de non-emploi, et plus particulièrement en période économique défavorable de contraction du marché de l'emploi. Tandis que les jeunes adultes concernés par la trajectoire 8, plus fréquemment des jeunes non diplômés ou peu diplômés CAP/BEP (certificat d'aptitude professionnelle et brevet d'études professionnelles), sont globalement dans une situation de chômage durable, sans recours à la formation. Néanmoins, pour une partie d'entre eux (Figure 5, en annexe de la version électronique de l'article), l'accès à l'emploi intervient après deux ans.

Le dernier groupe, trajectoire 2, le plus hétérogène, concerne des jeunes marqués par des trajectoires de précarité. Ils ont connu, soit des périodes importantes ou récurrentes de retour en études et formations au long de leur parcours, soit un chômage durable ou récurrent, ou encore une sortie d'emploi vers le chômage ou l'inactivité. Ce sont plus fréquemment des femmes (odds ratio $=1,95)$, des enfants de cadres (odds ratio $=1,52$ ) ou d'employés (odds ratio $=1,40)$, possédant un baccalauréat général $($ odds ratio $=1,68)$ et ils sont aussi plus fréquemment parents (18,2\% ont ou vont avoir des enfants).

Ainsi, dans ce groupe de jeunes adultes, nous retrouvons les trajectoires professionnelles spécifiques qui ont été mises en évidence par Virginie Mora (2014), à savoir celle de 
bacheliers, moins souvent issus de catégories sociales populaires, sortis de formation initiale et ayant entrepris une démarche de reprise d'études, après avoir connu des situations précédentes diverses, comme un emploi de courte durée, le chômage ou l'inactivité (Figure 5, en annexe de la version électronique de l'article). Le retour en formation de cette population est de plus en plus fréquent, puisqu'il concerne $30 \%$ de la Génération 2010, contre $24 \%$ de la Génération 2004 (Mora, Ibid.).

\section{Conclusion}

L’enquête Génération du Céreq, par sa qualité et son calendrier de l'insertion professionnelle et de l'autonomie résidentielle, permet d'analyser les trajectoires d'entrée dans la vie adulte des jeunes, grâce à la méthode d'analyse des séquences ou "optimal matching ". Nous avons ainsi pu déterminer huit trajectoires mesurant la fréquence des différentes combinaisons entre insertion professionnelle et autonomie résidentielle.

$42,5 \%$ des jeunes sortis du système scolaire à l'âge de vingt ans, en 2010, ont connu, au cours des trois années qui ont suivi, une trajectoire professionnelle plus ou moins précaire, et cela, quelle que soit la trajectoire résidentielle empruntée.

Si les conditions économiques ont rendu difficile le départ du foyer parental, le chômage n'est pas le seul facteur explicatif de la situation (Pan-Ké-Shon, 2010). D’autres facteurs, liés à l'immigration, aux conditions du marché locatif et à la situation économique des parents influencent négativement le processus d'indépendance et d'entrée dans l'âge adulte.

Si quitter le foyer parental n'est pas nécessairement synonyme d'émancipation financière, l'accès à l'emploi n'est pas davantage synonyme de décohabitation systématique. La crise économique impacte les trajectoires d'entrée dans la vie adulte des jeunes sortis du système scolaire en 2010, et les «voies de l'autonomie» (Van de Velde, 2015) sont multiples.

Ainsi, de jeunes adultes vont se positionner a contrario des situations " attendues " d'adéquation, entre trajectoire professionnelle et trajectoire d'autonomie résidentielle. Certains vont privilégier l'autonomie résidentielle, parfois associée à la constitution d'une famille, malgré la précarité professionnelle et, inversement, d'autres vont prolonger une cohabitation avec les parents, malgré une situation professionnelle et sociale favorable.

Le fait d'avoir apparié mensuellement les situations professionnelle et résidentielle a permis de révéler que la combinaison la plus fréquente (44,5\%) est «vivre chez les parents et être en emploi ». Or, ce n'est pas le lien qui paraissait le plus évident a priori, à l'inverse de ceux entre le "non-emploi et vivre chez les parents", et l' "emploi et la décohabitation". Cela confirme que l'accès à l'emploi durable n'est pas une condition suffisante au départ du foyer parental.

Cependant, la majorité $(57,5 \%)$ des sortants du système scolaire à vingt ans connaît une trajectoire professionnelle caractérisée par un accès rapide et durable à l'emploi. 
Trajectoires dont nous avons montré qu'elles étaient associées à un départ différé du foyer parental pour $12,2 \%$, un départ rapide et immédiat de ce même foyer pour $27,5 \%$ ou à un maintien durable au domicile des parents, dans la grande majorité des cas $(60,3 \%)$.

En conclusion, la relation entre autonomie résidentielle et insertion professionnelle n'est pas systématique quand on réalise une analyse des trajectoires. Un prolongement pertinent de ces travaux serait de travailler sur l'enquête Génération 2010 à cinq ans, pour mesurer d'éventuelles ruptures ou bifurcations dans les trajectoires. De plus, une analyse comparative avec une autre enquête Génération permettrait de mesurer les régularités et changements dans le temps, éventuellement liés à des contextes socioéconomiques différents.

\section{Bibliographie}

Billari F. (2001), "The analysis of early life course: complex descriptions of the transition to adulthood", Journal of Population Research, vol. 18 (2), Max Planck Institute for Démographic Research.

Castell L., Thouilleux C. \& Rivalin R. (2016), «L'accès à l'autonomie résidentielle pour les 18-24 ans : un processus socialement différencié ", Insee références, France, portrait social, pp. 10-25.

Driant J.C. (2016) "Les étudiants face à la crise du logement dans les territoires ", in Les vies étudiantes, tendances et inégalités, sous la direction de Giret J.-F., Van de Velde C., Verlet E, La Documentation française, pp. 191-209.

Galland O. (2011), Sociologie de la jeunesse, Paris, Armand Colin, Coll. U, 256 p.

Jauneau Y. (2007), «L'indépendance des jeunes adultes : chômeurs et inactifs cumulent les difficultés ", Insee première, $\mathrm{n}^{\circ} 1156,4 \mathrm{p}$.

Lelièvre E. \& Robette N, (2010), "Les trajectoires spatiales d'activité des couples ", Temporalités, $11 \mid 2010$.

Mora V., Sulzer E., Goffette C., Joseph O. (2008), «Insertion professionnelle et autonomie résidentielle des jeunes ", Les Travaux 2007-2008 de l'Observatoire national de la pauvreté et de l'exclusion sociale, Deuxième partie, Cahier 1, La Documentation française, pp. 291-313.

Mora V. (2014), «Quand les bacheliers reprennent des études - Qui, pourquoi, comment, quels effets sur l'insertion? "Net.Doc, n ${ }^{\circ} 127$, novembre, $102 \mathrm{p}$.

Orange S. (2013), L'autre enseignement supérieur. Les BTS et la gestion des aspirations scolaires, Presses universitaires de France, coll. «Education et Société », 208 p. 
Pan-Ké-Shon J.-L. (2010), « Partir de chez ses parents de 1968 à aujourd'hui », Idées, 162, pp. 33-41.

Portela M. \& Dezenaire F. (2014), «Quitter le foyer familial : les jeunes adultes confrontés à la crise économique ", Etudes et Résultats, DREES, nº 887, juillet 2014, 6 p.

Portela M., Saint Pol T. de, Albérola E. (2014), « Ressources et parcours vers l'indépendance des jeunes adultes en France. Associer soutien familial, aides publiques et revenus du travail ", Dossiers Solidarité et Santé, Drees, n 51.

Rault W. \& Régnier-Loilier A. (2015), «La première vie en couple : évolutions récentes », Population et sociétés, INED, $\mathrm{n}^{\circ}$ 521, avril, $4 \mathrm{p}$.

Régnier-Loilier A. (2011), "Situation résidentielle des étudiants et retour au foyer parental le week-end : une marche progressive vers l'indépendance ", in Galland O., Verley E. \& Vourch R. (dir.), Les mondes étudiants. Enquête conditions de vie 2010, Paris, La Documentation française, Coll. "Études \& Recherche », pp. 193-206.

Rémillon D. (2016), "L'analyse de la diversité des trajectoires professionnelles à partir de l'enquête Histoire de vie (2003) ", in Cordazzo Ph. \& Lelièvre E., Construire et analyser les trajectoires en démographie, Documents de travail, Ined, n²25, pp. 62-72.

Robette N. \& Thibault N. (2008), "Analyse harmonique qualitative ou méthodes d'appariement optimal ? Une analyse exploratoire des trajectoires professionnelles ", Population, (4), vol. 63, pp. 621-646.

Robette N. (2011), "Explorer et décrire les parcours de vie : les typologies de trajectoires ", Les clefs pour, CEPED, 80 p.

Robette N. (2012), L'analyse de séquences - Une introduction avec le logiciel $R$ et le package TraMineR. QUANTI/ Sciences sociales, accessible à http://quanti.hypotheses.org/686/.

Rouaud P., Joseph O. (coord.) (2014), Quand l'école est finie. Premiers pas dans la vie active de la génération 2010. Enquête 2013, Céreq, 92 p.

Van de Velde C. (2008), Devenir adulte. Sociologie comparée de la jeunesse en Europe, Paris, Presses universitaires de France, 278 p.

Van de Velde C. (2013), "Une jeunesse qui dure plus longtemps : une génération Tanguy? ", in L'état de la jeunesse en France, Alternatives économiques, Hors-série, Février, en partenariat avec Injep et Afev.

Van de Velde C. (2015), "Les voies de l'autonomie : les jeunes face à la crise en Europe ", Regards, $\mathrm{n}^{\circ}$ 48, octobre, pp. 81-93. 